\title{
Un résultat de trace pour les éléments finis de Crouzeix-Raviart, application à la discrétisation des équations de Darcy
}

\author{
par Christine Bernardi et Vivette Girault
}

Résumé: Les éléments finis de Crouzeix-Raviart engendrent un espace de fonctions discontinues, affines sur chaque élément d'une triangulation du domaine. Le but de cette note est de prouver un résultat de trace pour cet espace. On présente une application au traitement de conditions aux limites pour la discrétisation des équations de Darcy.

\begin{abstract}
Crouzeix-Raviart finite elements give rise to a space of discontinuous functions which are affine on each element of a triangulation of the domain. The aim of this note is to prove a trace result for this space. We present an application to the discretization of Darcy's equations.
\end{abstract}

Laboratoire Jacques-Louis Lions, C.N.R.S. \& Université Pierre et Marie Curie, B.C. 187, 4 place Jussieu, 75252 Paris Cedex 05, France. 



\section{Introduction.}

Soit $\Omega$ un ouvert borné connexe de $\mathbb{R}^{d}, d=2$ ou 3 , qui est soit un polygone $(d=2)$ soit un polyèdre à frontière lipschitzienne $(d=3)$. On introduit une famille régulière de triangulations $\left(\mathcal{T}_{h}\right)_{h}$ de $\Omega$, au sens usuel $[4, \S 17], h$ désignant le maximum des diamètres des éléments de $\mathcal{T}_{h}$. L'espace associé aux éléments finis introduits par M. Crouzeix et P.-A. Raviart dans [5] est

$$
\mathbb{Y}_{h}=\left\{q_{h} \in L^{2}(\Omega) \text { affine sur chaque } K \in \mathcal{T}_{h} \text { et continu en tout point de } \mathcal{V}_{h}\right\}
$$

où $\mathcal{V}_{h}$ désigne l'ensemble des milieux de chaque arête $(d=2)$ ou barycentres de chaque face $(d=3)$ des éléments de $\mathcal{T}_{h}$. Les fonctions de $\mathbb{Y}_{h}$ sont en général discontinues et n'appartiennent donc à aucun espace $H^{s}(\Omega)$ pour $s \geq \frac{1}{2}$. En outre, leurs traces n'appartiennent pas à $H^{\frac{1}{2}}(\partial \Omega)$. On ne peut donc pas leur appliquer le théorème de trace usuel. Le but principal de cette note est d'énoncer un résultat de trace plus faible et de le démontrer par des arguments de dualité. On réfère à [3] pour d'autres propriétés de l'espace $\mathbb{Y}_{h}$.

En dépit de leur manque de conformité et parce qu'ils sont localement conservatifs, les éléments finis de Crouzeix-Raviart sont utilisés pour la discrétisation d'un grand nombre de problèmes issus de la mécanique des fluides, par exemple les équations de Navier-Stokes ou de Darcy. On explique ici comment le résultat de trace sert à l'analyse du système de Darcy, lorsque l'espace $\mathbb{Y}_{h}$ est utilisé pour la pression selon un schéma suggéré dans $[1, \S 4]$. 


\section{Le résultat de trace et sa démonstration.}

L'espace $\mathbb{Y}_{h}$ est muni de la semi-norme et norme brisées:

$$
|q|_{H_{h}^{1}(\Omega)}=\left(\sum_{K \in \mathcal{T}_{h}}|q|_{H^{1}(K)}^{2}\right)^{\frac{1}{2}}, \quad\|q\|_{H_{h}^{1}(\Omega)}=\left(\|q\|_{L^{2}(\Omega)}^{2}+|q|_{H_{h}^{1}(\Omega)}^{2}\right)^{\frac{1}{2}} .
$$

Les normes des espaces $H^{\sigma}(\partial \Omega), 0<\sigma<1$, sont définies par interpolation.

Théorème 1. Pour tout nombre réel $\sigma, 0 \leq \sigma<\frac{1}{2}$, il existe une constante $c_{\sigma}$ indépendante de $h$ telle que

$$
\forall q_{h} \in \mathbb{Y}_{h}, \quad\left\|q_{h}\right\|_{H^{\sigma}(\partial \Omega)} \leq c_{\sigma}\left\|q_{h}\right\|_{H_{h}^{1}(\Omega)}
$$

Démonstration. En quatre étapes.

1) Préliminaires

Nous supposons que $0<\sigma<\frac{1}{2}$. Pour tout élément $q_{h}$ de $\mathbb{Y}_{h}$, on utilise la décomposition

$$
q_{h}=\tilde{q}_{h}+\bar{q}, \quad \text { avec } \quad \bar{q}=\frac{1}{\operatorname{mes}(\Omega)} \int_{\Omega} q_{h}(\boldsymbol{x}) d \boldsymbol{x} .
$$

Il est évident que

$$
\|\bar{q}\|_{H^{\sigma}(\partial \Omega)} \leq\left(\frac{\operatorname{mes}(\partial \Omega)}{\operatorname{mes}(\Omega)}\right)^{\frac{1}{2}}\left\|q_{h}\right\|_{L^{2}(\Omega)}
$$

La majoration de $\left\|\tilde{q}_{h}\right\|_{H^{\sigma}(\partial \Omega)}$ est basée sur l'argument de dualité

$$
\left\|\tilde{q}_{h}\right\|_{H^{\sigma}(\partial \Omega)}=\sup _{\varphi \in H^{-\sigma}(\partial \Omega)} \frac{\left\langle\varphi, \tilde{q}_{h}\right\rangle}{\|\varphi\|_{H^{-\sigma}(\partial \Omega)}}
$$

où $\langle\cdot, \cdot\rangle$ désigne le produit de dualité entre $H^{-\sigma}(\partial \Omega)$ et $H^{\sigma}(\partial \Omega)$. Pour tout $\varphi$ dans $H^{-\sigma}(\partial \Omega)$, le problème

$$
\begin{cases}-\Delta \mu=-\frac{1}{\operatorname{mes}(\Omega)}\langle\varphi, 1\rangle & \text { dans } \Omega \\ \partial_{n} \mu=\varphi & \text { sur } \partial \Omega\end{cases}
$$

admet une solution unique dans $H^{\frac{3}{2}-\sigma}(\Omega) \cap L_{0}^{2}(\Omega)$, où $L_{0}^{2}(\Omega)$ désigne l'espace des fonctions de $L^{2}(\Omega)$ d'intégrale nulle sur $\Omega$. Cette solution vérifie en outre, pour une constante $c$ ne dépendant que de $\Omega$,

$$
\|\mu\|_{H^{\frac{3}{2}-\sigma}(\Omega)} \leq c\|\varphi\|_{H^{-\sigma}(\partial \Omega)} .
$$

Comme $\tilde{q}_{h}$ est d'intégrale nulle sur $\Omega$, on obtient par intégration par parties

$$
\left\langle\varphi, \tilde{q}_{h}\right\rangle=\sum_{K \in \mathcal{T}_{h}} \int_{K}(\operatorname{grad} \mu)(\boldsymbol{x}) \cdot\left(\operatorname{grad} \tilde{q}_{h}\right)(\boldsymbol{x}) d \boldsymbol{x}+\sum_{e \in \mathcal{E}_{h}^{0}}\left\langle\partial_{n} \mu,\left[\tilde{q}_{h}\right]_{e}\right\rangle_{e}
$$

où $\mathcal{E}_{h}^{0}$ désigne l'ensemble des côtés $(d=2)$ ou faces $(d=3)$ d'éléments de $\mathcal{T}_{h}$ qui ne sont pas contenues dans $\partial \Omega$. La notation $[\cdot]_{e}$ représente le saut à travers $e$ avec le signe approprié 
et $\langle\cdot, \cdot\rangle_{e}$ désigne le produit de dualité restreint à $e$. Le premier terme se majore grâce à une inégalité de Cauchy-Schwarz

$$
\left|\sum_{K \in \mathcal{T}_{h}} \int_{K}(\operatorname{grad} \mu)(\boldsymbol{x}) \cdot\left(\operatorname{grad} \tilde{q}_{h}\right)(\boldsymbol{x}) d \boldsymbol{x}\right| \leq|\mu|_{H^{1}(\Omega)}\left|\tilde{q}_{h}\right|_{H_{h}^{1}(\Omega)} .
$$

2) Évaluation of $\left\langle\partial_{n} \mu,\left[\tilde{q}_{h}\right]_{e}\right\rangle_{e}$

On note $\bar{q}_{e}$ la moyenne de $\tilde{q}_{h}$ sur $e$ et on pose $\rho_{e}=\frac{1}{\operatorname{mes}(e)}\left\langle\partial_{n} \mu, 1\right\rangle_{e}$. On voit que

$$
\left\langle\partial_{n} \mu,\left[\tilde{q}_{h}\right]_{e}\right\rangle_{e}=\left\langle\partial_{n} \mu-\rho_{e},\left[\tilde{q}_{h}-\bar{q}_{e}\right]_{e}\right\rangle_{e}
$$

On suppose e partagé par deux éléments $K$ et $K^{\prime}$ de $\mathcal{T}_{h}$, de sorte que

$$
\left|\left\langle\partial_{n} \mu,\left[\tilde{q}_{h}\right]_{e}\right\rangle_{e}\right| \leq\left\|\partial_{n} \mu-\rho_{e}\right\|_{H^{-\sigma}(e)}\left(\left\|\tilde{q}_{h \mid K}-\bar{q}_{e}\right\|_{H^{\sigma}(e)}+\left\|\tilde{q}_{h \mid K^{\prime}}-\bar{q}_{e}\right\|_{H^{\sigma}(e)}\right) .
$$

Par passage à l'élément de référence $\hat{\kappa}$ et en utilisant une inégalité de Bramble-Hilbert, on obtient pour $\kappa$ égal à $K$ ou à $K^{\prime}$ et avec les notations usuelles

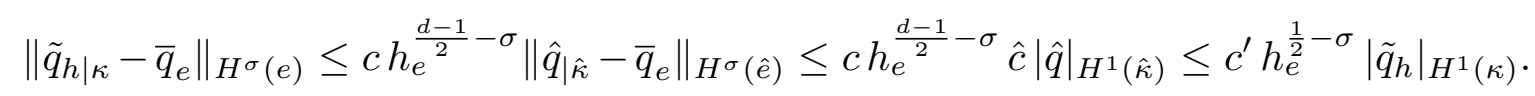

En combinant (10) et (11), on déduit

$$
\left|\left\langle\partial_{n} \mu,\left[\tilde{q}_{h}\right]_{e}\right\rangle_{e}\right| \leq c h_{e}^{\frac{1}{2}-\sigma}\left\|\partial_{n} \mu-\rho_{e}\right\|_{H^{-\sigma}(e)}\left(\left|\tilde{q}_{h}\right|_{H^{1}(K)}+\left|\tilde{q}_{h}\right|_{H^{1}\left(K^{\prime}\right)}\right) .
$$

3) Évaluation of $\left\|\partial_{n} \mu-\rho_{e}\right\|_{H^{-\sigma}(e)}$

On a

$$
\left\|\partial_{n} \mu-\rho_{e}\right\|_{H^{-\sigma}(e)}=\sup _{\chi \in H^{\sigma}(e)} \frac{\left\langle\partial_{n} \mu-\rho_{e}, \chi\right\rangle_{e}}{\|\chi\|_{H^{\sigma}(e)}} .
$$

Si $\bar{\chi}_{e}$ désigne la moyenne de $\chi$ sur $e$, on déduit du choix de $\rho_{e}$ que

$$
\left\langle\partial_{n} \mu-\rho_{e}, \chi\right\rangle_{e}=\left\langle\partial_{n} \mu, \chi-\bar{\chi}_{e}\right\rangle_{e}
$$

Soit $\chi^{*}$ le relèvement (construit à partir d'un opérateur de relèvement fixé sur un élément de référence $\hat{K}$ à valeurs dans $L_{0}^{2}(\hat{K})$ ) de l'extension par zéro de $\chi-\bar{\chi}_{e}$ à $\partial K$ (on utilise ici le fait que $\sigma<\frac{1}{2}$ ). Comme $\Delta \mu$ est constant sur $K$ et $\chi^{*}$ appartient à $L_{0}^{2}(K)$, on a

$$
\left\langle\partial_{n} \mu, \chi-\bar{\chi}_{e}\right\rangle_{e}=\int_{K}(\operatorname{grad} \mu)(\boldsymbol{x}) \cdot\left(\operatorname{grad} \chi^{*}\right)(\boldsymbol{x}) d \boldsymbol{x},
$$

d'où

$$
\left\langle\partial_{n} \mu, \chi-\bar{\chi}_{e}\right\rangle_{e} \leq\|\mu\|_{H^{\frac{3}{2}-\sigma}(K)}\left\|\operatorname{grad} \chi^{*}\right\|_{H^{\sigma-\frac{1}{2}}(K)^{d}} .
$$

Le dernier terme se majore par

$$
\left\|\operatorname{grad} \chi^{*}\right\|_{H^{\sigma-\frac{1}{2}}(K)^{d}}=\sup _{\psi \in H^{\frac{1}{2}-\sigma}(K)^{d}} \frac{\left\langle\operatorname{grad} \chi^{*}, \boldsymbol{\psi}\right\rangle_{K}}{\|\boldsymbol{\psi}\|_{H^{\frac{1}{2}-\sigma}(K)^{d}}} .
$$


On constate aussi que grad $\chi^{*}$ est orthogonal aux constantes sur $K$. Par suite, en désignant par $\overline{\boldsymbol{\psi}}_{K}$ la moyenne de $\boldsymbol{\psi}$ sur $K$, on obtient par passage à l'élément de référence $\hat{K}$ (grâce à un argument de régularisation) et en notant que le gradient est continu de $H^{\sigma+\frac{1}{2}}(\hat{K})$ dans $H^{\sigma-\frac{1}{2}}(\hat{K})$,

$$
\left\langle\operatorname{grad} \chi^{*}, \boldsymbol{\psi}\right\rangle_{K} \leq c^{\prime} h_{K}^{d-1}\left\|\hat{\chi}^{*}\right\|_{H^{\sigma+\frac{1}{2}(\hat{K})}}\left\|\hat{\boldsymbol{\psi}}-\overline{\boldsymbol{\psi}}_{K}\right\|_{H^{\frac{1}{2}-\sigma}(\hat{K})^{d}} .
$$

Des inégalités

$$
\begin{aligned}
& \left\|\hat{\chi}^{*}\right\|_{H^{\sigma+\frac{1}{2}(\hat{K})}} \leq c\left\|\hat{\chi}-\bar{\chi}_{e}\right\|_{H^{\sigma}(\hat{e})} \leq c^{\prime}|\hat{\chi}|_{H^{\sigma}(\hat{e})} \leq c^{\prime \prime} h_{K}^{\sigma-\frac{d-1}{2}}|\chi|_{H^{\sigma}(e)}, \\
& \left\|\hat{\boldsymbol{\psi}}-\overline{\boldsymbol{\psi}}_{K}\right\|_{H^{\frac{1}{2}-\sigma}(\hat{K})^{d}} \leq c|\hat{\boldsymbol{\psi}}|_{H^{\frac{1}{2}-\sigma}(\hat{K})^{d}} \leq c^{\prime} h_{K}^{\frac{1}{2}-\sigma-\frac{d}{2}}\|\boldsymbol{\psi}\|_{H^{\frac{1}{2}-\sigma}(K)^{d}}
\end{aligned}
$$

on déduit

$$
\left\|\operatorname{grad} \chi^{*}\right\|_{H^{\sigma-\frac{1}{2}}(K)^{d}} \leq c\|\chi\|_{H^{\sigma}(e)},
$$

d'où

$$
\left\|\partial_{n} \mu-\rho_{e}\right\|_{H^{-\sigma}(e)} \leq c\|\mu\|_{H^{\frac{3}{2}-\sigma}(K)} \cdot
$$

4) Conclusion

En insérant (13) dans (12) et en combinant le résultat avec (6) à (9), on obtient

$$
\left\|\tilde{q}_{h}\right\|_{H^{\sigma}(\partial \Omega)} \leq c\left|\tilde{q}_{h}\right|_{H_{h}^{1}(\Omega)}
$$

On note que les semi-normes $|\cdot|_{H_{h}^{1}(\Omega)}$ de $q_{h}$ et $\tilde{q}_{h}$ coïncident et on déduit le résultat cherché de (4), (5) et (14).

Remarque. Les arguments de la démonstration précédente, combinés avec [2, Rem. 2.10], indiquent que la constante $c_{\sigma}$ dans (3) se comporte comme $c^{\prime}\left(\frac{1}{2}-\sigma\right)^{-1}$ lorsque $\sigma$ tend vers $\frac{1}{2}$. 


\section{Une discrétisation par éléments finis des équations de Darcy.}

Les équations de Darcy modélisent l'écoulement laminaire d'un fluide visqueux incompressible dans un milieu poreux:

$$
\begin{cases}\alpha \boldsymbol{u}+\operatorname{grad} p=\mathbf{0} & \operatorname{dans} \Omega, \\ \operatorname{div} \boldsymbol{u}=0 & \operatorname{dans} \Omega, \\ \boldsymbol{u} \cdot \boldsymbol{n}=k & \operatorname{sur} \partial \Omega\end{cases}
$$

Les inconnues sont la vitesse $\boldsymbol{u}$ et la pression $p$ du fluide. La donnée $k$ représente la vitesse du fluide en entrée et sortie du domaine et $\alpha$ est un tenseur symétrique défini positif lié à la viscosité du fluide et à la perméabilité du milieu, que l'on suppose constant pour simplifier.

Le système (15) admet plusieurs formulations variationnelles équivalentes. Nous avons choisi de travailler avec la suivante

$$
\begin{aligned}
& \text { Trouver }(\boldsymbol{u}, p) \text { dans } L^{2}(\Omega)^{d} \times\left(H^{1}(\Omega) \cap L_{0}^{2}(\Omega)\right) \text { tel que } \\
& \qquad \boldsymbol{v} \in L^{2}(\Omega)^{d}, \quad \int_{\Omega} \alpha \boldsymbol{u}(\boldsymbol{x}) \cdot \boldsymbol{v}(\boldsymbol{x}) d \boldsymbol{x}+\int_{\Omega} \boldsymbol{v}(\boldsymbol{x}) \cdot(\operatorname{grad} p)(\boldsymbol{x}) d \boldsymbol{x}=0, \\
& \forall q \in H^{1}(\Omega) \cap L_{0}^{2}(\Omega), \quad \int_{\Omega} \boldsymbol{u}(\boldsymbol{x}) \cdot(\operatorname{grad} q)(\boldsymbol{x}) d \boldsymbol{x}=\langle k, q\rangle .
\end{aligned}
$$

Il est facile de vérifier que, pour toute donnée $k$ dans $H^{-\frac{1}{2}}(\partial \Omega)$ vérifiant $\langle k, 1\rangle=0$, ce problème admet une solution unique.

La discrétisation que nous présentons maintenant a été proposée dans $[1, \S 4]$. Soit $\mathbb{X}_{h}$ l'espace des fonctions de $L^{2}(\Omega)^{d}$ dont la restriction à chaque élément de $\mathcal{T}_{h}$ est constante sur $K$. On pose

$$
\mathbb{M}_{h}=\left\{q_{h} \in \mathbb{Y}_{h} ; \int_{\Omega} q_{h}(\boldsymbol{x}) d \boldsymbol{x}=0\right\}
$$

En désignant par $\mathcal{E}_{h}^{\partial \Omega}$ l'ensemble des côtés $(d=2)$ ou faces $(d=3)$ d'éléments de $\mathcal{T}_{h}$ qui sont contenues dans $\partial \Omega$, on introduit une approximation $k_{h}$ de $k$ :

$$
\forall e \in \mathcal{E}_{h}^{\partial \Omega}, \quad k_{h \mid e}=\frac{1}{\operatorname{mes}(e)}\langle k, 1\rangle_{e}
$$

On note que la définition précédente requiert que $k$ appartienne à $H^{-\sigma}(\partial \Omega), \sigma<\frac{1}{2}$. Le problème discret s'écrit

$$
\begin{aligned}
& \text { Trouver }\left(\boldsymbol{u}_{h}, p_{h}\right) \text { dans } \mathbb{X}_{h} \times \mathbb{M}_{h} \text { tel que } \\
& \qquad \boldsymbol{v}_{h} \in \mathbb{X}_{h}, \quad \int_{\Omega} \alpha \boldsymbol{u}_{h}(\boldsymbol{x}) \cdot \boldsymbol{v}_{h}(\boldsymbol{x}) d \boldsymbol{x}+\int_{\Omega} \boldsymbol{v}_{h}(\boldsymbol{x}) \cdot\left(\operatorname{grad} p_{h}\right)(\boldsymbol{x}) d \boldsymbol{x}=0 \\
& \forall q_{h} \in \mathbb{M}_{h}, \quad \int_{\Omega} \boldsymbol{u}_{h}(\boldsymbol{x}) \cdot\left(\operatorname{grad} q_{h}\right)(\boldsymbol{x}) d \boldsymbol{x}=\left\langle k_{h}, q_{h}\right\rangle
\end{aligned}
$$


Remarque. Une des conséquences du choix (18) de $k_{h}$ est que le problème (19) est parfaitement équivalent au système

$$
\begin{cases}\alpha \boldsymbol{u}_{h}+\operatorname{grad} p_{h}=\mathbf{0} & \operatorname{dans} K, K \in \mathcal{T}_{h}, \\ \operatorname{div} \boldsymbol{u}_{h}=0 & \text { dans } \Omega, \\ \boldsymbol{u}_{h} \cdot \boldsymbol{n}=k_{h} & \text { sur } \partial \Omega\end{cases}
$$

donc à un schéma de volumes finis conservatif.

L'ellipticité de la forme: $\left(\boldsymbol{u}_{h}, \boldsymbol{v}_{h}\right) \mapsto \int_{\Omega} \alpha \boldsymbol{u}_{h}(\boldsymbol{x}) \cdot \boldsymbol{v}_{h}(\boldsymbol{x}) d \boldsymbol{x}$ sur $\mathbb{X}_{h}$ est évidente. En outre, en prenant $\boldsymbol{v}_{h}$ tel que, sur chaque $K, \boldsymbol{v}_{h \mid K}=\operatorname{grad} q_{h \mid K}$, on démontre la condition inf-sup suivante, pour une constante $\beta$ indépendante de $h$ (voir [1, Lemma 4.2] pour plus de détails)

$$
\forall q_{h} \in \mathbb{M}_{h}, \quad \sup _{\boldsymbol{v}_{h} \in \mathbb{X}_{h}} \frac{\int_{\Omega} \boldsymbol{v}_{h}(\boldsymbol{x}) \cdot\left(\operatorname{grad} q_{h}\right)(\boldsymbol{x}) d \boldsymbol{x}}{\left\|\boldsymbol{v}_{h}\right\|_{L^{2}(\Omega)^{d}}} \geq \beta\left\|q_{h}\right\|_{H_{h}^{1}(\Omega)} .
$$

En combinant ces propriétés avec le Théorème 1, on obtient le résultat suivant.

Proposition 2. Pour toute donnée $k$ dans $H^{-\sigma}(\partial \Omega), \sigma<\frac{1}{2}$, vérifiant $\langle k, 1\rangle=0$, le problème (19) admet une solution unique $\left(\boldsymbol{u}_{h}, p_{h}\right)$ dans $\mathbb{X}_{h} \times \mathbb{M}_{h}$. En outre, il existe une constante $c$ indépendante de $h$ telle que

$$
\left\|\boldsymbol{u}_{h}\right\|_{L^{2}(\Omega)^{d}}+\left\|p_{h}\right\|_{H_{h}^{1}(\Omega)} \leq c\left\|k_{h}\right\|_{H^{-\sigma}(\partial \Omega)}
$$

Les propriétés précédentes permettent également de majorer l'erreur entre les solutions $(\boldsymbol{u}, p)$ et $\left(\boldsymbol{u}_{h}, p_{h}\right)$ en fonction de la distance de $(\boldsymbol{u}, p)$ à $\mathbb{X}_{h} \times \mathbb{M}_{h}$, d'un terme d'erreur de consistance et, grâce au Théorème 1, de l'erreur d'approximation $\left\|k-k_{h}\right\|_{H^{-\sigma}(\partial \Omega)}$. En choisissant $\sigma=\frac{1}{2}-\frac{1}{|\log h|}$ et en utilisant l'évaluation de $c_{\sigma}$ donnée en remarque, on en déduit le résultat suivant.

Proposition 3. On suppose la donnée $k$ dans $H^{\rho}(\partial \Omega), 0 \leq \rho \leq 1$, et la solution $(\boldsymbol{u}, p)$ du problème (16) dans $H^{s}(\Omega)^{d} \times H^{s+1}(\Omega), 0<s \leq 1$. On a alors la majoration d'erreur a priori entre cette solution et la solution $\left(\boldsymbol{u}_{h}, p_{h}\right)$ du problème (19)

$$
\begin{aligned}
\left\|\boldsymbol{u}-\boldsymbol{u}_{h}\right\|_{L^{2}(\Omega)^{d}} & +\left\|p-p_{h}\right\|_{H_{h}^{1}(\Omega)} \\
& \leq c\left(h^{\rho+\frac{1}{2}}|\log h|\|k\|_{H^{\rho}(\partial \Omega)}+h^{s}\left(\|\boldsymbol{u}\|_{H^{s}(\Omega)^{d}}+\|p\|_{H^{s+1}(\Omega)}\right)\right) .
\end{aligned}
$$

On note la convergence de la discrétisation même pour une donnée très peu régulière. 


\section{Références bibliographiques}

[1] Achdou Y., Bernardi C., Coquel F., A priori and a posteriori analysis of finite volume discretizations of Darcy's equations, Numer. Math. 96 (2003), 17-42.

[2] Bertoluzza S., Perrier V., The mortar method in the wavelet context, Modél. Math. Anal. Numér. 35 (2001), 647-673.

[3] Brenner S.C., Poincaré-Friedrichs inequalities for piecewise $H^{1}$ functions, SIAM J. Numer. Anal. 41 (2003), 306-324.

[4] Ciarlet P.G., Basic Error Estimates for Elliptic Problems, Handbook of Numerical Analysis, Vol. II, édité par P.G. Ciarlet \& J.-L. Lions (1989).

[5] Crouzeix M., Raviart P.-A., Conforming and nonconforming finite element methods for solving the stationary Stokes equations, R.A.I.R.O. Anal. Numér. 7 R3 (1973), $33-76$. 


\section{Abridged English Version}

\section{A trace result for nonconforming Crouzeix-Raviart finite elements, application to the discretization of Darcy's equations}

\section{Introduction}

Let $\Omega$ be a bounded connected domain in $\mathbb{R}^{d}, d=2$ or 3 , which is either a polygon $(d=2)$ or a polyhedron with a Lipschitz-continuous boundary $(d=3)$, and let $\left(\mathcal{T}_{h}\right)_{h}$ be a regular family of triangulations of $\Omega$ by triangles or tetrahedra (see $[4, \S 17]$ ), where $h$ stands for the maximum of the diameters of the elements of $\mathcal{T}_{h}$. The space associated with the finite elements introduced by M. Crouzeix and P.-A. Raviart in [5] is the space defined in (1), i.e. the space $\mathbb{Y}_{h}$ of functions $q_{h}$ in $L^{2}(\Omega)$ such that

- their restrictions to each element $K$ of $\mathcal{T}_{h}$ is affine on $K$,

- they are continuous at the midpoint of each edge $(d=2)$ or at the barycenter of each face $(d=3)$ of elements of $\mathcal{T}_{h}$.

It can be noted that such functions do not belong in general to any Sobolev space $H^{s}(\Omega)$ for $s \geq \frac{1}{2}$. So the standard trace theorem cannot be applied to $\mathbb{Y}_{h}$. The aim of this note is to prove a weaker trace result by duality arguments.

The Crouzeix-Raviart finite elements are used in the discretization of a number of flow problems, for instance the Navier-Stokes system or Darcy's equations, particularly because they are locally conservative. Our trace result is an important tool for the analysis of Darcy's equations when the space $\mathbb{Y}_{h}$ is used for the discrete pressures, as suggested in $[1, \S 4]$. Other properties of the space $\mathbb{Y}_{h}$ can be found in [3].

\section{The trace result}

The space $\mathbb{Y}_{h}$ is usually equipped with the broken seminorm and norm introduced in (2). The norms of the spaces $H^{\sigma}(\partial \Omega), 0<\sigma<1$, are defined by interpolation.

Theorem 1. For any real number $\sigma, 0 \leq \sigma<\frac{1}{2}$, there exists a constant $c_{\sigma}$ independent of $h$ such that

$$
\forall q_{h} \in \mathbb{Y}_{h}, \quad\left\|q_{h}\right\|_{H^{\sigma}(\partial \Omega)} \leq c_{\sigma}\left\|q_{h}\right\|_{H_{h}^{1}(\Omega)}
$$

The proof of this result uses the splitting (4). The norm of the trace of the second term of this sum is bounded in (5). We use a duality argument to evaluate the analogue for the first part, see (6). Next, technical arguments lead to estimate (14). Combining (5) and (14) gives the desired result.

\section{A finite element discretization of Darcy's equations}

Darcy's equations (15) model the laminar flow of a viscous incompressible fluid in a porous medium. The unknowns are the velocity $\boldsymbol{u}$ and the pressure $p$ of the fluid. The 
datum $k$ represents the inward and outward flows and $\alpha$ is a positive-definite symmetric tensor which is assumed to be constant for simplicity and depends on the viscosity of the fluid and the permeability of the medium. Equations (15) admit among others the equivalent variational formulation (16) and have a unique solution for any datum $k$ in $H^{-\frac{1}{2}}(\partial \Omega)$ satisfying $\langle k, 1\rangle=0$.

To describe the discrete problem, we introduce the space $\mathbb{X}_{h}$ of functions which are constant on each element $K$ of $\mathcal{T}_{h}$, together with the space $\mathbb{M}_{h}$ in (17). Assuming that the datum $k$ belongs to $H^{-\sigma}(\partial \Omega), \sigma<\frac{1}{2}$, we define an approximation $k_{h}$ of $k$ in (18). This leads to the discrete problem (19). It can be noted that, thanks to this choice of $k_{h}$, it is equivalent to the finite volume scheme (20).

We refer to $[1, \S 4]$ for the numerical analysis of problem (19). More precisely, the discrete inf-sup condition (21) results from [1, Lemma 4.2]. Combining this with Theorem 1 , we derive the next results.

Proposition 2. For any datum $k$ in $H^{-\sigma}(\partial \Omega), \sigma<\frac{1}{2}$, satisfying $\langle k, 1\rangle=0$, problem (19) admits a unique solution which moreover satisfies (22).

Proposition 3. Assume that the datum $k$ belongs to $H^{\rho}(\partial \Omega), 0 \leq \rho \leq 1$, and that the solution $(\boldsymbol{u}, p)$ of problem (16) belongs to $H^{s}(\Omega)^{d} \times H^{s+1}(\Omega), 0<s \leq 1$. The error estimate (23) holds between this solution and the solution $\left(\boldsymbol{u}_{h}, p_{h}\right)$ of problem (19). 